\title{
In-depth Proteomic Quantification of Cell Secretome in Se- rum-Containing Conditioned Medium
}

Yejing Weng ${ }^{\dagger, \star}$, Zhigang Sui ${ }^{\dagger}$, Yichu Shan ${ }^{\dagger}$, Hao Jiang ${ }^{\dagger, *}$, Yuan Zhou ${ }^{\dagger}$, Xudong Zhu ${ }^{\dagger, \star}$, Zhen Liang ${ }^{\dagger}$, Lihua Zhang, ${ }^{\dagger}, *$ and Yukui Zhang ${ }^{\dagger}$

${ }^{\dagger}$ Key Lab of Separation Sciences for Analytical Chemistry, National Chromatographic R. \& A. Center, Dalian Institute of Chemical Physics, Chinese Academy of Sciences, Dalian 116023, China.

${ }^{t}$ University of Chinese Academy of Sciences, Beijing 100049, China.

\section{RESULTS}

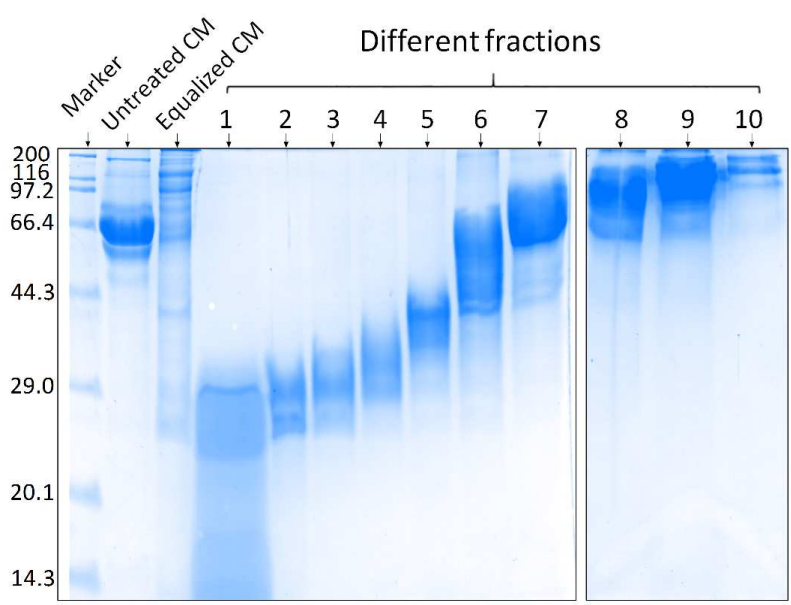

Figure S1. SDS-PAGE profiling of untreated CM, equalized CM and ten fractions separated by GELFrEE system. CM: conditioned medium.

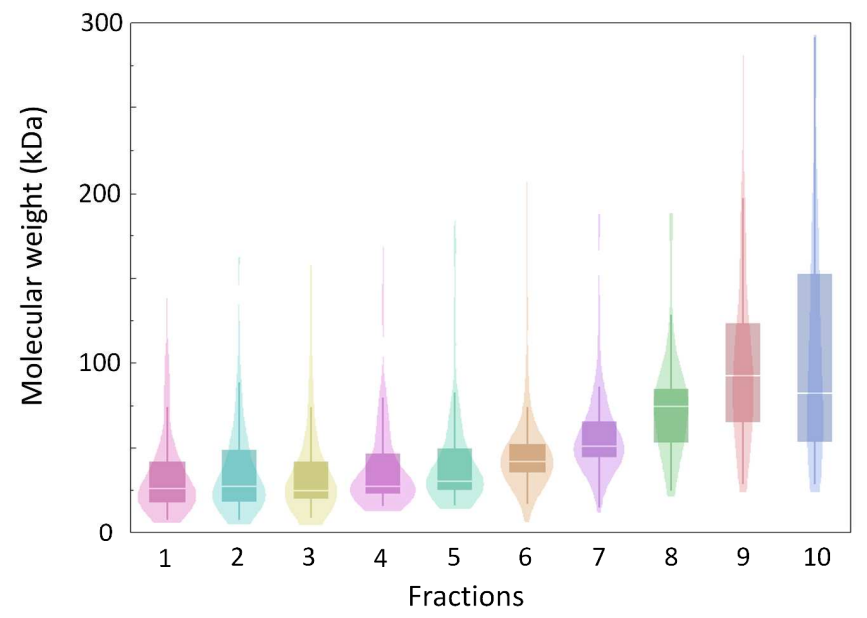

Figure S2. Violin plots of Mw distribution of identified proteins in different fractions. Violin shape, kernel density curves; white lines in boxplot, median; the boxplots show the span from 25th to 75th percentile. 